\title{
Prevalencia de la disfagia secundaria al tratamiento de cáncer de cabeza y
cuello
}

\section{En una cohorte de una institución en Bogotá}

Prevalence of dysphagia secondary to the treatment of head and neck cancer in a cohort of an institution in Bogotá: In a cohort of an institution in Bogotá

(c) $($ ) $\$(9)$

Jorge Iván Marco Antonio Castañeda Maldonado Andrea Marcela Suárez Velásquez
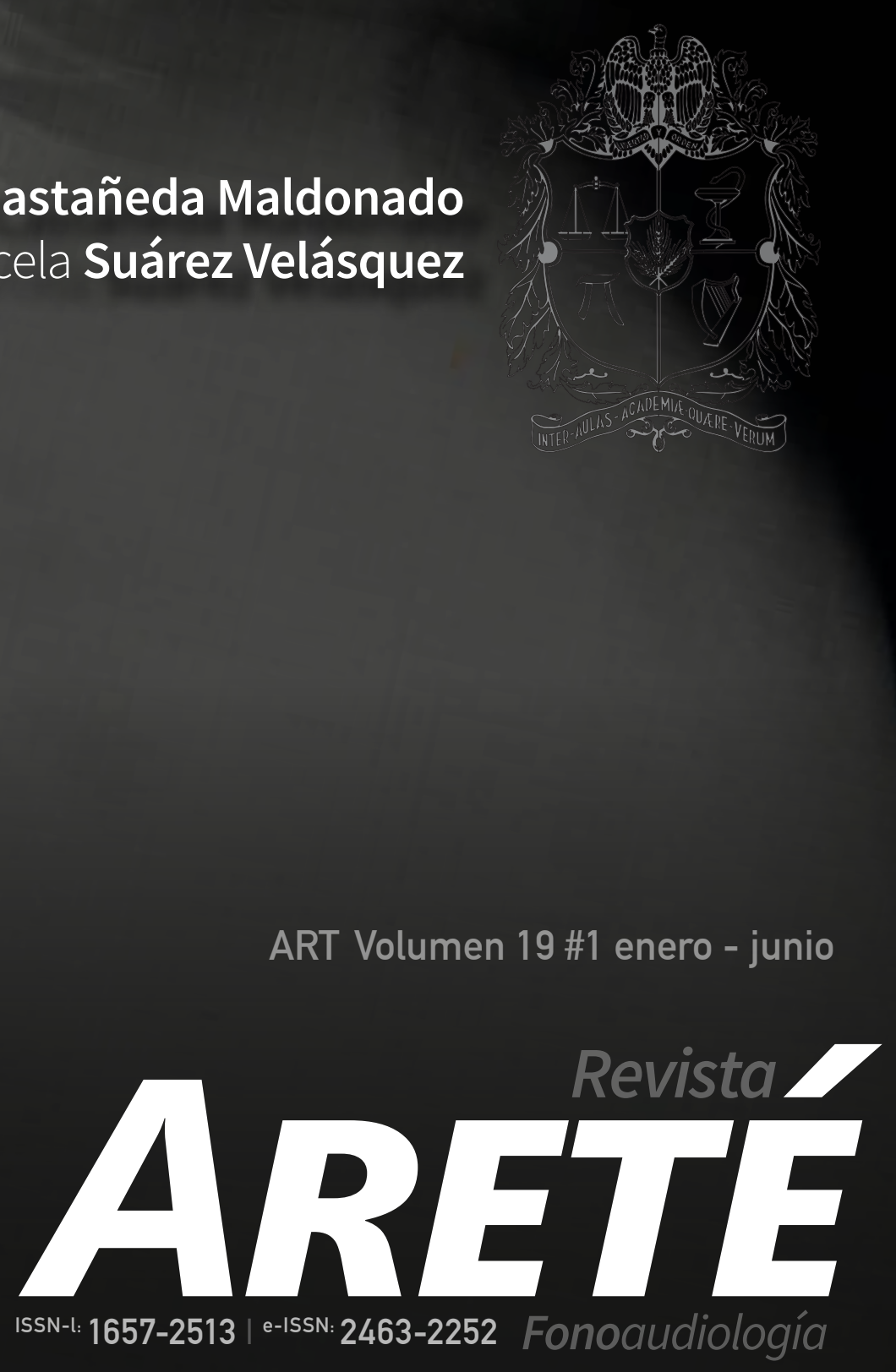


\section{$A R E^{\text {Revestion }}$ ARETÉ \\ 1657-2513 | e-ISSN: 2463-2252 Fonoaudiología}

ID:

1657-2513.art.19106

Title: $\quad$ Prevalence of dysphagia secondary to the treatment of head and neck cancer

Subtitle: In a cohort of an institution in bogotá

Título: $\quad$ Prevalencia de la disfagia secundaria al tratamiento de cáncer de cabeza y cuello

Subtítulo: En una cohorte de una institución en bogotá

Alt Title / Título alternativo:

[en]: $\quad$ Prevalence of dysphagia secondary to head and neck cancer treatment In a cohort of an institution in Bogotá

[es]:Prevalencia de la disfagia secundaria al tratamiento de cáncer de cabeza y cuello En una cohorte de una institución en Bogotá

Author (s) / Autor (es):

Castañeda Maldonado, \& Suárez Velásquez

Keywords / Palabras Clave:

[en]: Dysphagia, head and neck cancer, speech therapy, radiotherapy, chemotherapy, head and neck surgery.

[es]: Disfagia, cáncer de cabeza y cuello, fonoaudiología, radioterapia, quimioterapia, cirugía de cabeza y cuello

Submited: $\quad$ 2019-05-27

Acepted: $\quad$ 2015-07-29
Resumen

La incidencia del cáncer de cabeza y cuello ha aumentado en los últimos años. Los tratamientos como la quimioradioterapia, han tenido efectividad en su manejo, sin embargo, se evidencian secuelas

como la disfagia. El presente estudio pretende

estimar la prevalencia de pacientes con disfagia orofaríngea secundaria al tratamiento de cáncer de cabeza y cuello atendidos en una institución de cuarto nivel en Bogotá Colombia, y describir el perfil del paciente con cáncer de cabeza y cuello que presenta

disfagia orofaríngea en esta institución. Se realizó un estudio retrospectivo transversal de prevalencia en disfagia orofaríngea reportada en el servicio de consulta externa de una institución de cuarto nivel especializada en atención a pacientes oncológicos. Se revisaron las historias clínicas reportadas de agosto a octubre del año 2016, se incluyeron pacientes mayores

de 3 años, con diagnóstico subjetivo de disfagia orofaríngea según Escala FOIS (Funtional Oral Intake Scale). Se identificaron 56 pacientes de los cuales 36 presentaban disfagia orofaríngea según la evaluación clínica. La edad media fue de 57,1 años de los cuales el 58,3\% eran mujeres. Estos pacientes a través de su proceso oncológico recibieron diferentes tratamientos médicos en diferentes combinaciones. Ocho pacientes

lograron la rehabilitación de la disfagia. No se evidenció correlación estadística entre las variables sexo y tipo de cáncer mediante test $X^{2}(P<0.014)$. Se concluye que la disfagia es un efecto significativo secundario al tratamiento de cáncer de cabeza y cuello con una prevalencia del 64,28\% en la muestra seleccionada con mayor frecuencia en mujeres. La recuperación exitosa de la disfagia no depende de factores independientes de edad y sexo, evidenciando más influencia de la modalidad de tratamiento oncológico.

\section{Citar como:}

Castañeda Maldonado, J. I., \& Suárez Velásquez, A. M. (2019). Prevalencia de la disfagia secundaria al tratamiento de cáncer de cabeza y cuello: En una cohorte de una institución en bogotá. Areté, 19 (1), [pgln]-[pgOut]. Obtenido de: https://arete.ibero.edu.co/article/view/1353
Head and neack cancer have increased. The chemoradiotherapy treatment is effective for the cancer managment. However, there are consequences such as dysphagia. To estimate the prevalence of patients with oropharyngeal dysphagia secondary to head and neck cancer treated in a fourth level institution in Bogotá -

Colombia, and to describe the patient profile with head and neck cancer that presents oropharyngeal dysphagia within this institution. A retrospective cross-sectional study of prevalence in oropharyngeal dysphagia was reported in the external query service of a fourth-level institution specialized in cancer care. The reported medical charts were took from auqust to october of 2016

were patients older than 3 years and with a subjective diagnosis of oropharyngeal dysphagia according to the Oral Intake Scale Funtional Oral scale (FOIS) were included. 56 patients were identified, 36 of whom had oropharyngeal dysphagia according to the clinical evaluation.

The average age was 57.1 years of which 58.3\% were women. These patients, through their cancer process, received different medical treatments in different combinations. Eight patients achieved

rehabilitation of dysphagia. There was no statistical correlation between the variables sex and type of cancer by means of the $\nabla^{2}$ test (P \&lt;0.014). It is concluded that dysphagia is a significant effect secondary to the treatment of head and neck cancer with a prevalence of $64.28 \%$ in the sample most frequently selected in women.

The successful recovery of dysphagia does not depend on independent factors of age and sex, showing more influence of the modality of cancer treatment.
Jorge Iván Marco Antonio Castañeda Maldonado, Msc $\mathrm{BSH}$

\section{Source | Filiacion:}

Universidad Nacional de Colombia

\section{BIO:}

Fonoaudiólogo de la Universidad Nacional de Colombia M.Sc Investigación Clínica.

City | Ciudad:

Bogotá DC [co]

e-mail:

jimacm17@gmail.com
Andrea Marcela Suárez Velásquez, BSH

Source | Filiacion:

Universidad Nacional de Colombia

BIO:

Fonoaudióloga de la Universidad Nacional de Colombia M.Sc Morfología (Estudiante).

\section{City | Ciudad:}

Bogotá DC [co]

e-mail:

amsuarezvelas@gmail.com 


\section{Prevalencia de la disfagia secundaria al tratamiento de cáncer de cabeza y cuello}

En una cohorte de una institución en Bogotá

Prevalence of dysphagia secondary to the treatment of head and neck cancer: In a cohort of an institution in Bogotá

Jorge Iván Marco Antonio Castañeda Maldonado Andrea Marcela Suárez Velásquez

\section{Introducción}

El cáncer de cabeza y cuello es una patología que ha ido en aumento tanto a nivel mundial como en Colombia. Según la OMS, para el año 2016, han sido reportados más de 630000 casos al año (Cárcamo, 2018).

Los tipos de cáncer de cabeza y cuello se clasifican topográficamente en: cáncer de cavidad bucal, orofaringe, nasal, seno paranasal, nasofaringe, laringe e hipofaringe (American Cancer Society, NHC., 2015). Existe otro tipo de cáncer que, aunque no aparece en esta clasificación, genera alteraciones importantes en procesos de comunicación y deglución, conocido como cáncer de tiroides (Greenblatt, y otros, 2009) (Nickel, y otros, 2019).Desde el tratamiento médico-oncológico se cuentan con varias opciones enfocadas tanto a la curación como al manejo paliativo. Dentro de estos tratamientos hay tres grandes grupos: la cirugía, la radioterapia y la quimioterapia, que según el tiempo, estadio, tamaño y extensión puede ser neoadyuvante (tratamiento inicial), adyuvante (tratamiento complementario) o coadyuvante (tratamiento concomitante) (American Cancer Society, NHC., 2015) (Cadena, Guerra, \& Pérez-Mitchell, 2014) (European Society for Medical Oncology, 2015). 


\section{Prevalencia de la disfagia secundaria al tratamiento de cáncer de cabeza y cuello} En una cohorte de una institución en bogotá

La resección quirúrgica incluye extirpación local del tumor en los primeros estadios, la resección con reconstrucción primaria o cirugía conservadora se hace necesaria en estadios avanzados y en tumores ubicados en la región posterior de la cavidad oral en donde además existe la posibilidad de extraer ganglios linfáticos y se tiene como objetivo la protección de estructuras sanas (Smeele, 2014); en los casos más severos se requiere cirugía reconstructiva mediante la implantación de colgajos, bien sea radial, pectoral o antero lateral del muslo, el objetivo es reestablecer la apariencia y la función de los tejidos extirpados (European Society for Medical Oncology, 2015).Otra modalidad de tratamiento es la radioterapia que utiliza ondas de alta energía, tales como los rayos $X$, rayos gamma, rayos de electrones o de protones, para eliminar o dañar las células cancerosas. La radiación actúa sobre el ADN que se encuentra dentro de las células produciendo pequeñas roturas que evitan que las células cancerosas crezcan y se dividan. Puede que también las células normales cercanas se afecten con la radiación, pero la mayoría se recupera y vuelve a tener una función normal (American Cancer Society, NHC., 2015).La radioterapia es usualmente utilizada como tratamiento adyuvante a la resección quirúrgica de cáncer de cabeza y cuello para controlar la afección microscópica (European Society for Medical Oncology, 2015).La quimioterapia se suministra a través de vía endovenosa y actúa sistémicamente. Existen varios medicamentos que pueden combinarse entre ellos (cisplatino, 5-fluoracilo, docetaxel, entre otros), o ser utilizados en coadyuvancia con la radioterapia potenciando el tratamiento, ya que actúa interrumpiendo el proceso de reproducción descontrolada de algunas células cancerosas (Lyndell, 2014) (European Society for Medical Oncology, 2015).

Estos tratamientos que interrumpen el proceso de crecimiento y división de las células cancerosas, también lo hacen en las células sanas y por lo tanto generan lesión en los tejidos adyacentes. Los efectos secundarios de los tratamientos en cáncer de cabeza y cuello influyen en procesos como el habla y la deglución. Las personas sometidas a cirugía de cualquiera de las modalidades pueden presentar disfagia por el daño/resección muscular, óseo, cartilaginoso, o de estructuras nerviosas, así como por la eliminación de aponeurosis del cuello. La gravedad del déficit de la deglución es dependiente del tamaño y la localización de la lesión, y el grado y la extensión de la resección quirúrgica. El edema, dolor, cicatrización y lesión nerviosa debido a la disección del cuello son todas las causas potenciales en la patogénesis de la disfunción para deglutir (Denaro, Merlano, \& Russi, 2013). El procedimiento de reconstrucción seleccionado después de la resección del cáncer oral, de orofaringe o laringe también juega un papel importante en las habilidades de habla y deglución; la reconstrucción con colgajos produce ausencia de sensibilidad y reducción en la velocidad y coordinación del movimiento (Logemann, Pauloski, Rademaker, \& Colangelo, 1997).

Por otro lado, la radiación clásica preoperativa ha sido utilizada para reducir el tamaño del tumor primario priorizándolo sobre la cirugía; esta modalidad ha sido reemplazada con la quimio-radioterapia (Pauloski, 2008). El objetivo principal de tratamiento no quirúrgico es la preservación de la anatomía y función de respiración, deglución y comunicación en el cáncer de cabeza y cuello, sin embargo, se ha probado que esta preservación anatómica no significa necesariamente una preservación funcional (Santini, Robert, Lagier, Dessi, \& Fakhry, 2015). Fibrosis, xerostomía, odinofagia, mucositis, necrosis en tejidos blandos y osteoradionecrosis de la mandíbula, pérdida de peso y la necesidad de uso de técnicas alternativas de alimentación son efectos secundarios que terminan en trastornos de la deglución afectando la eficiencia y seguridad en la ingesta (Portas, y otros, 2011) (Szczesniak, Maclean, Zhang, Graham, \& Cook, 2014).

La quimioterapia en sí misma genera efectos como náuseas y vómito ya que afectan el centro sensitivo vagal, y puede irritar la mucosa orofaríngea generando odinofagia. La mucositis se está relacionada inicialmente con el agente 5-desoxyuridylate (Shaw \& Martino, 2013) (Joung, Woo, Beom, Hwa, \& Kyu, 2015). La neurotoxicidad y ototoxicidad es producida en su mayoría por las drogas alcalinas generando déficit en la sinapsis lo cual disminuye el rango de movilidad por la actividad neuromuscular (Lyndell, 2014).

Se podría decir entonces, que la disfagia puede iniciar en la fase pretratamiento que en un principio es tipo mecánica (Martínez \& Bellido, 2003) por la alteración estructural y modificación en el espacio oral o faríngeo que repercute directamente en el tránsito del bolo. Después del tratamiento oncológico las estructuras cambian al igual que su funcionalidad, aquí la disfagia ya no es solo mecánica sino mixta ya que el componente motor entra en juego aumentando la incapacidad de deglutir.

Los signos de disfagia más comunes incluyen un incremento de tiempo para el tránsito oral, con habilidad reducida para manejo del bolo en la cavidad oral, acumulación de residuo oral y en faringe, rango de movilidad lingual reducido, movilidad hioidea reducida, disminución en la estabilidad glótica, aspiración, disminución en movilidad de base de lengua y pared faríngea, disfunción cricofaríngea (Lazarus, Ward, \& Yiu, 2014), disminución de la peristalsis faríngea y reducción del tiempo de contacto de la base lingual con la pared posterior faríngea (Pauloski, 2008) (Hamilton, y otros, 2008), lo que genera malnutrición, deshidratación, pérdida de peso, habilidades funcionales reducidas, y miedo al comer, estos factores pueden incidir en la depresión y reducir la calidad de vida (Zhang, Huang, Wu, Chen, \& Huang, 2014).

El presente artículo da a conocer la prevalencia de la disfagia secundaria a los tratamientos de cáncer de cabeza y cuello basado en la recolección de datos por historias clínicas en un periodo de tres meses, además se describe el perfil del paciente con cáncer de cabeza y cuello manejado desde la profesión de fonoaudiología.

\section{Metodología}

\section{Diseño y población de estudio}

Se diseñó un estudio unicéntrico retrospectivo transversal de prevalencia de disfagia orofaríngea en consulta externa de una institución de cuarto nivel especializada en el manejo de pacientes con patología oncológica en la ciudad de Bogotá. Se incluyeron personas de todas las edades ingresadas en el servicio de fonoaudiología que hayan tenido al menos tres sesiones de intervención, con diagnóstico oncológico primario de cabeza y cuello. Se excluyeron pacientes de hospitalización, unidad de cuidados intensivos y urgencias.

\section{Selección de la muestra y recogida de datos}

Se realizó un análisis a partir de la historia clínica de cada paciente registrada en esta institución. Se elaboró una lista de recolección de datos de manera online que se corroboró por parte de ambos autores de manera separada. Una vez identificados los pacientes con disfagia orofaríngea como diagnóstico inicial según FOIS, en su primera sesión (evaluación) se registraron los datos de relevancia clínica escogidos por los autores y basados en búsqueda de literatura. 


\section{Análisis estadístico.}

Los datos se analizaron mediante el paquete estadístico JAMOVi ${ }^{\circledR}$ para Windows. Se efectuó análisis descriptivo con medidas de proporción para variables cualitativas y medidas de tendencia central para variables cuantitativas usándose intervalo de confianza (IC) del 95\% correspondiente.

Las principales variables a evaluar fueron edad, sexo, topografía de cáncer, tratamiento oncológico, el tratamiento fonoaudiológico, número de sesiones fonoaudiológicas, diagnóstico final de disfagia. Los tratamientos fonoaudiológicos se establecieron de manera dicotómica buscando facilitar el análisis estadístico; se tomó como -SI- uso de la maniobra cuando ésta se usaba al menos en una de las sesiones de intervención fonoaudiológica. El diagnóstico fonoaudiológico de disfagia también fue dicotomizado; se tomó como -SI- diagnóstico de disfagia orofaríngea cuando la calificación según Fols es 5 o menor a través de la evaluación clínica subjetiva de la disfagia. Las variables edad y número de sesiones fonoaudiológicas dicotomizaron por la mediana. Se realizaron análisis para relación de dependencia entre dos variables mediante el test de $\chi^{2} y$ Test de Fisher. No se realizaron otros estudios por el limitado número de pacientes.

\section{Resultados}

Se identificaron 56 pacientes del centro de atención especializada en cáncer entre los meses agosto y octubre del año 2016 por el servicio de Fonoaudiología, de los cuales 36 (64,28\%) tenían el diagnóstico de Disfagia Orofaríngea y fueron incluidos en el análisis.

Los 36 pacientes incluidos en el análisis tienen una edad media y desviación estándar de 57,1 (19,2) años respectivamente (mínimo de 3 años y máximo de 87 años). El 58,3\% eran mujeres, siendo la edad media 53,4 años (inferior a la de los hombres 62,2 años), con un comportamiento de distribución no normal (Shapiro Wilk: 0.897) frente a la encontrada en los hombres que mantenía una distribución normal (Shapiro Wilk: <0.0001). La distribución por edad y sexo se muestra en la ilustración 1.

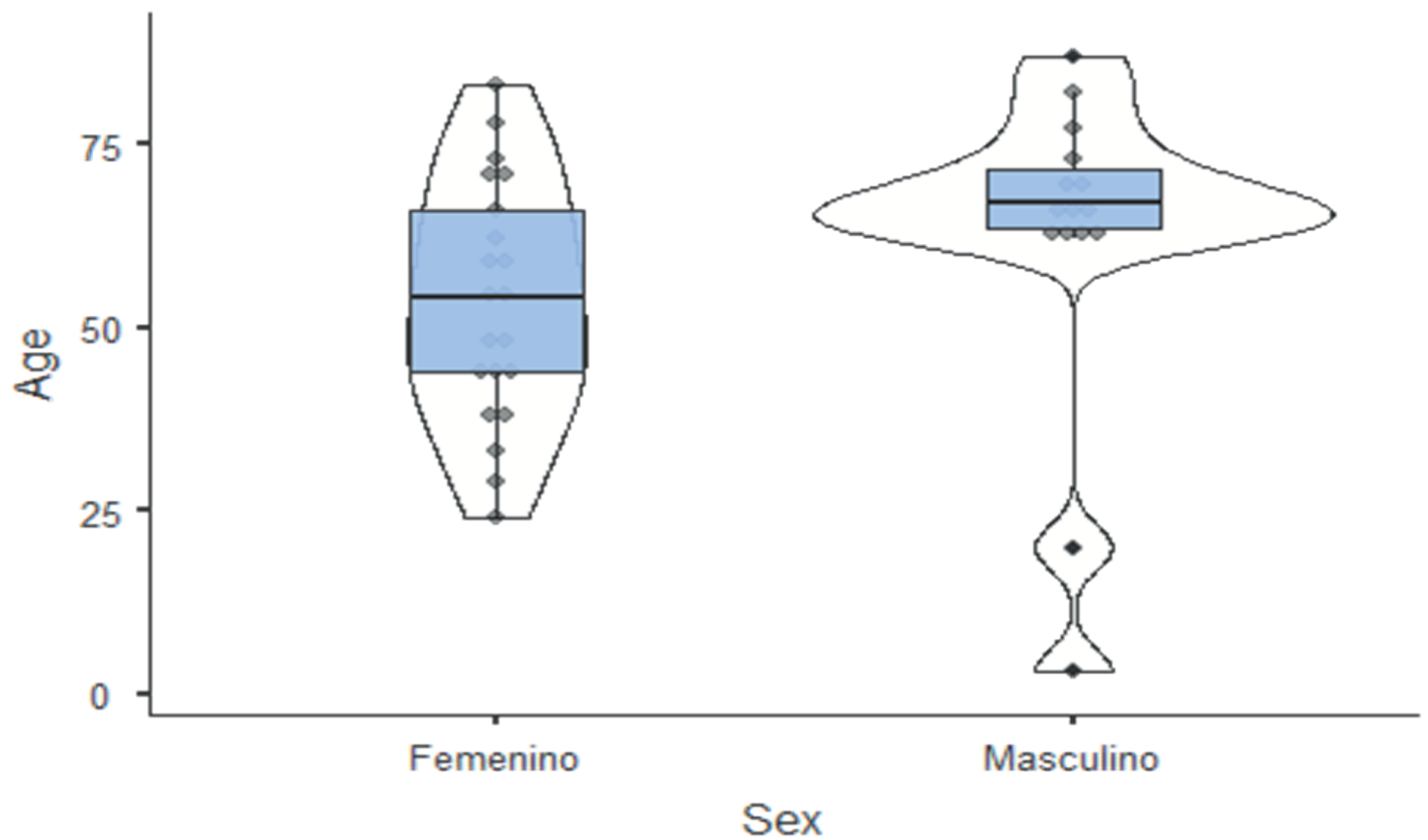

Fuente: elaboración propia del autor

Los tipos de cáncer encontrados en esta población de estudio se muestran en la Tabla 1, se evidenció una relación (femenino:masculino) importante en los cánceres de tiroides (8:1), mandíbula (6:0), y larin- ge (0:6). La rehabilitación de la disfagia (según escala FOIS) según tipo de cáncer se puede ver en la Gráfica 1.

Tabla 1. Frecuencia en tipo de cáncer según el sexo en los pacientes con diagnóstico de disfagia orofaríngea en una institución de Bogotá.

\begin{tabular}{|c|c|c|c|c|c|c|c|c|c|c|}
\hline \multicolumn{11}{|c|}{ Frecuencias según Sexo } \\
\hline \multicolumn{11}{|c|}{ Tipo de Cáncer - N - } \\
\hline Sexo & Tiroides & Parótida & Orofaringe & Oral & Nasofaringe & Maxilar & Mandíbula & Lengua & Laringe & Cuello \\
\hline Femenino & 8 & 1 & 2 & 2 & 0 & 2 & 6 & 1 & 0 & 0 \\
\hline Masculino & 1 & 0 & 1 & 2 & 1 & 1 & 0 & 1 & 6 & 1 \\
\hline
\end{tabular}


Prevalencia de la disfagia secundaria al tratamiento de cáncer de cabeza y cuello

En una cohorte de una institución en bogotá

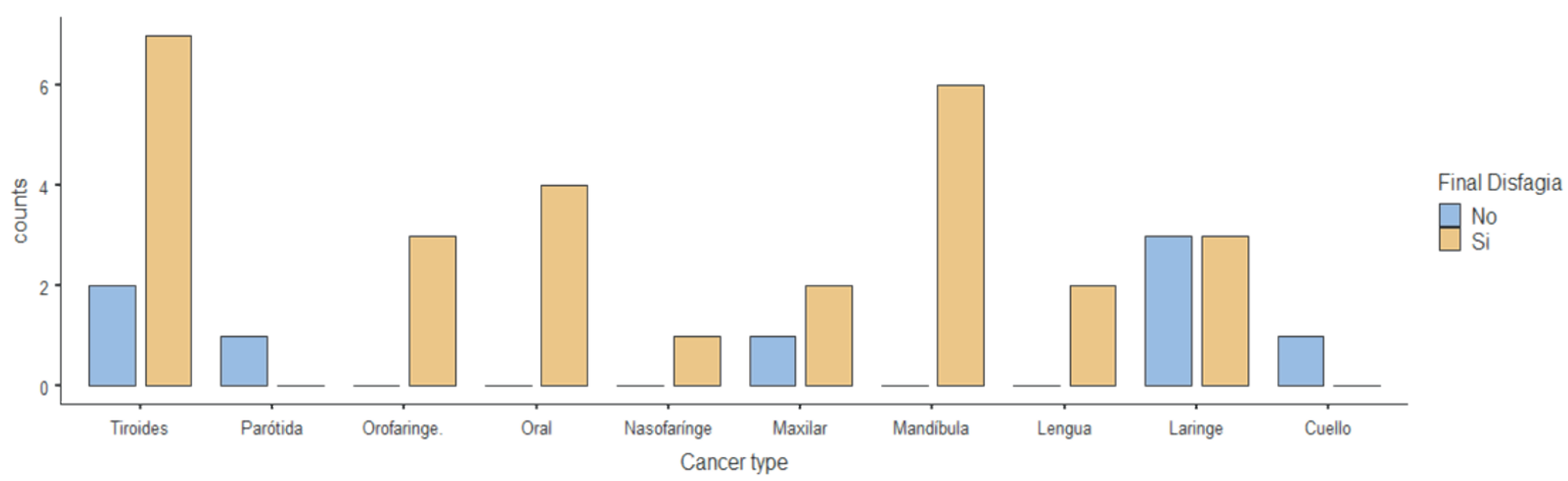

Gráfica 1. Resultados de rehabilitación de la disfagia según tipo de cáncer.

Se realizaron 334 sesiones de Fonoaudiología para los 36 pacientes, con una media de 9,28 y mediana de 9,50 sesiones. El mínimo de sesiones fue 3 y el máximo 24. El número de sesiones realizadas a los ocho pacientes que lograron la rehabilitación completa tenía una me- dia de 8,00 (+-4,14) en comparación con los 28 pacientes que no la lograron y requirieron 9,64 (+-5,77). El múmero de sesiones que tomaron las personas que superaron su condición de disfagia se puede ver en la llustración 2.

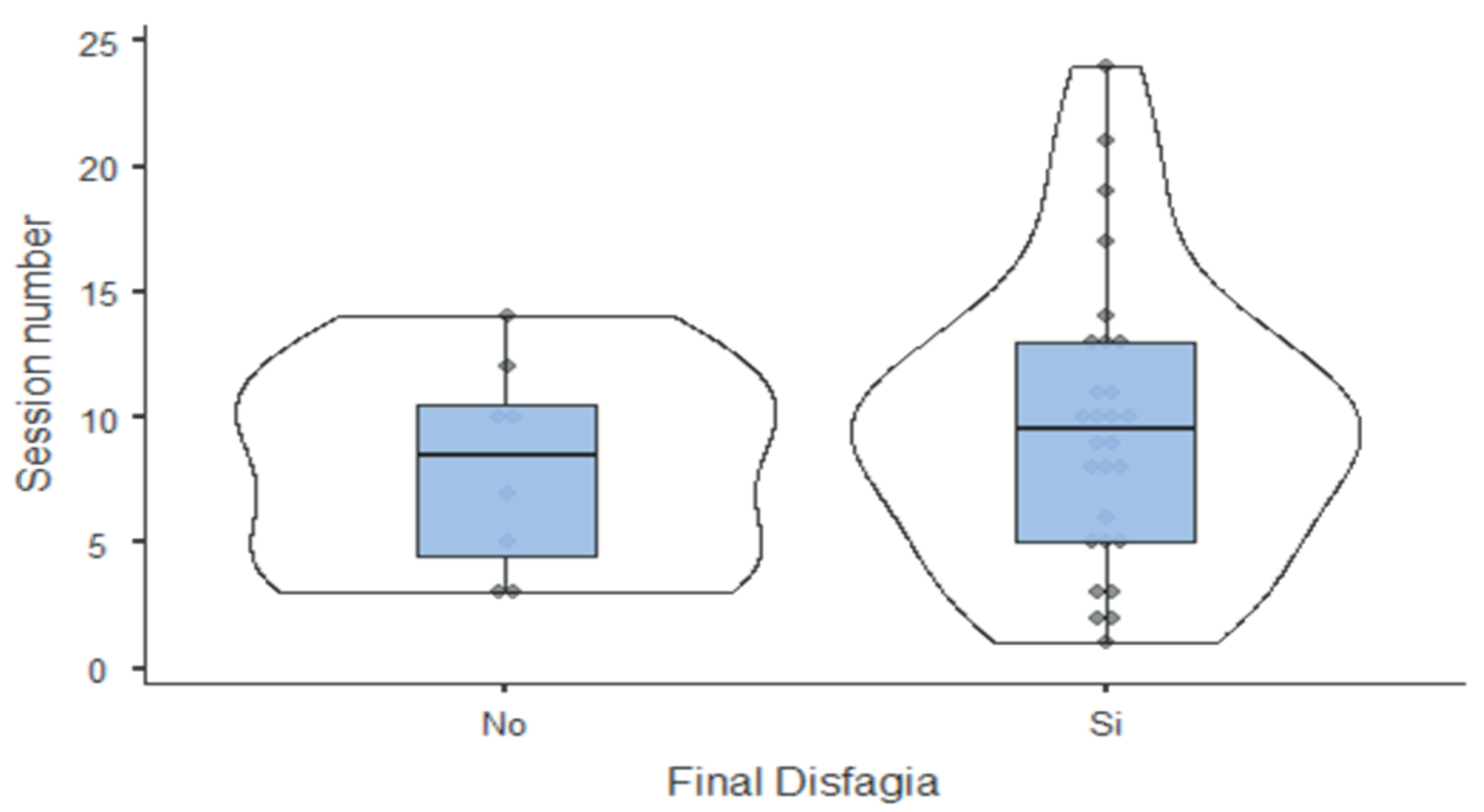

Ilustración 2. Número de sesiones requeridas para los pacientes que superaron la disfagia.

Fuente: elaboración propia del autor

Las combinaciones de los tratamientos médicos se pueden evidenciar en la Tabla 2. La mayoría de pacientes estuvieron sometidos a un $(30,6 \%)$ o dos $(47,2 \%)$ tratamientos. Ocho pacientes previamen- te habían pasado por radioterapia, cirugía y quimioterapia durante su proceso de cáncer. 
Tabla 2. Combinaciones en los tratamientos médicos en los pacientes con cáncer de cabeza y cuello en una institución de Bogotá.

\begin{tabular}{lc}
\multicolumn{1}{c}{$\mathbf{N}^{\circ}$ de tratamientos médicos } & $\mathbf{N}^{\circ}$ de Pacientes (\%) \\
Un tratamiento & $11(30,6 \%)$ \\
Radioterapia & 1 \\
Cirugía & 9 \\
Quimioterapia & 1 \\
\hline Dostratamientos & $11(47,2 \%)$ \\
\hline Radioterapia + Cirugía & 1 \\
\hline Cirugía + Quimioterapia & 2 \\
\hline Radioterapia + Quimioterapia & 3 \\
\hline Radioterapia + Yodoterapia & $8(22,2 \%)$ \\
\hline Trestratamientos & 7 \\
\hline Radioterapia + Cirugía + Quimioterapia & 1 \\
\hline Radioterapia + Cirugía + Yodoterapia & 36 \\
\hline
\end{tabular}

Fuente: Elaboración propia del autor.

El número de tratamientos médicos que recibe cada paciente en relación a su rehabilitación de disfagia se puede apreciar en el Gráfico

2. Es importante recalcar que de los pacientes con cáncer de tiroides que recibieron tres tratamientos médicos, ninguno logró la rehabilitación de su condición de disfagia.

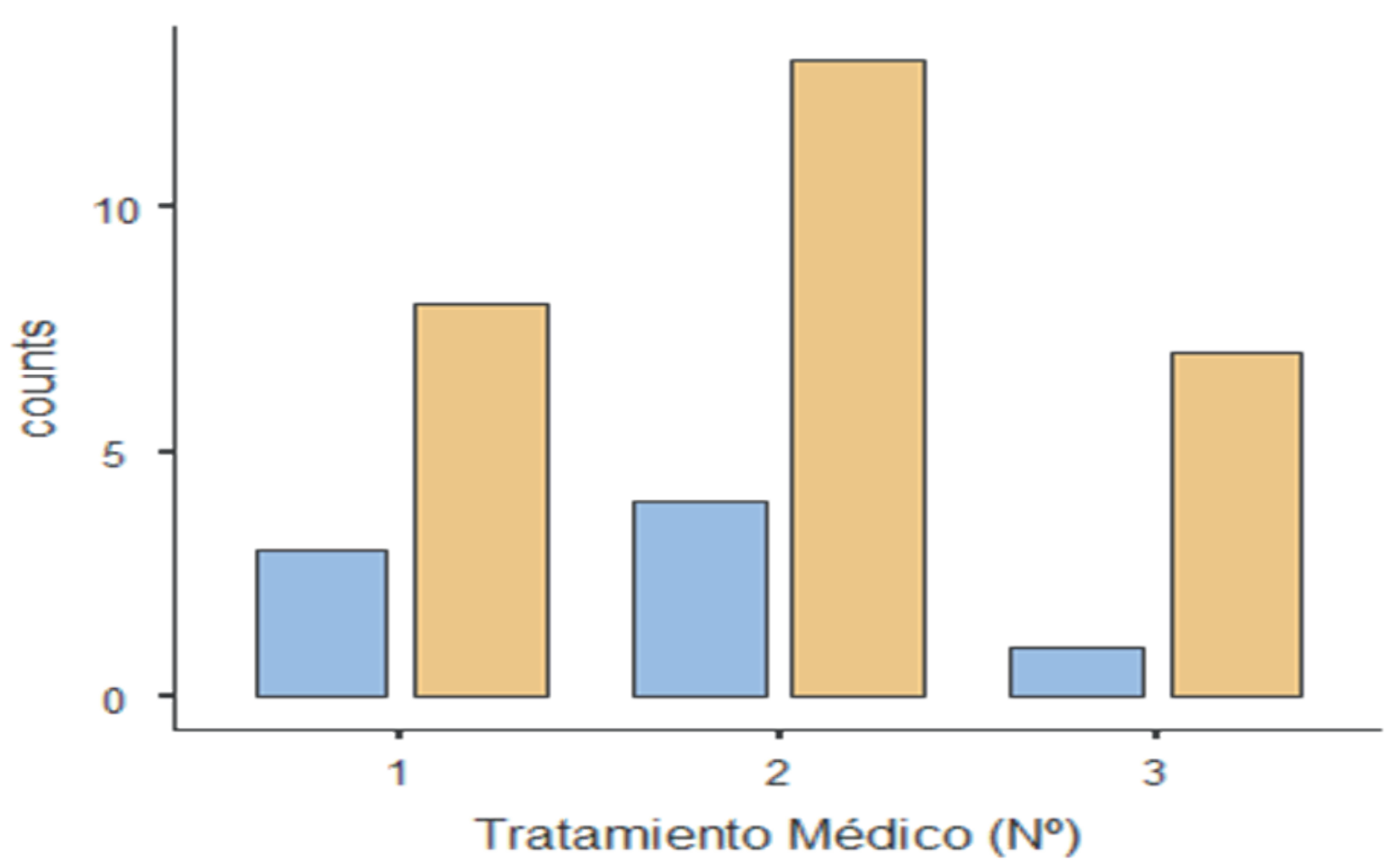

\section{Final Disfagia}

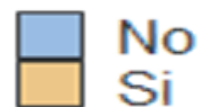

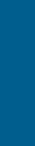




\section{Prevalencia de la disfagia secundaria al tratamiento de cáncer de cabeza y cuello}

En una cohorte de una institución en bogotá

Tabla 3. Frecuencias en tratamientos fonoaudiológicos para pacientes con cáncer de cabeza y cuello en una institución de Bogotá.

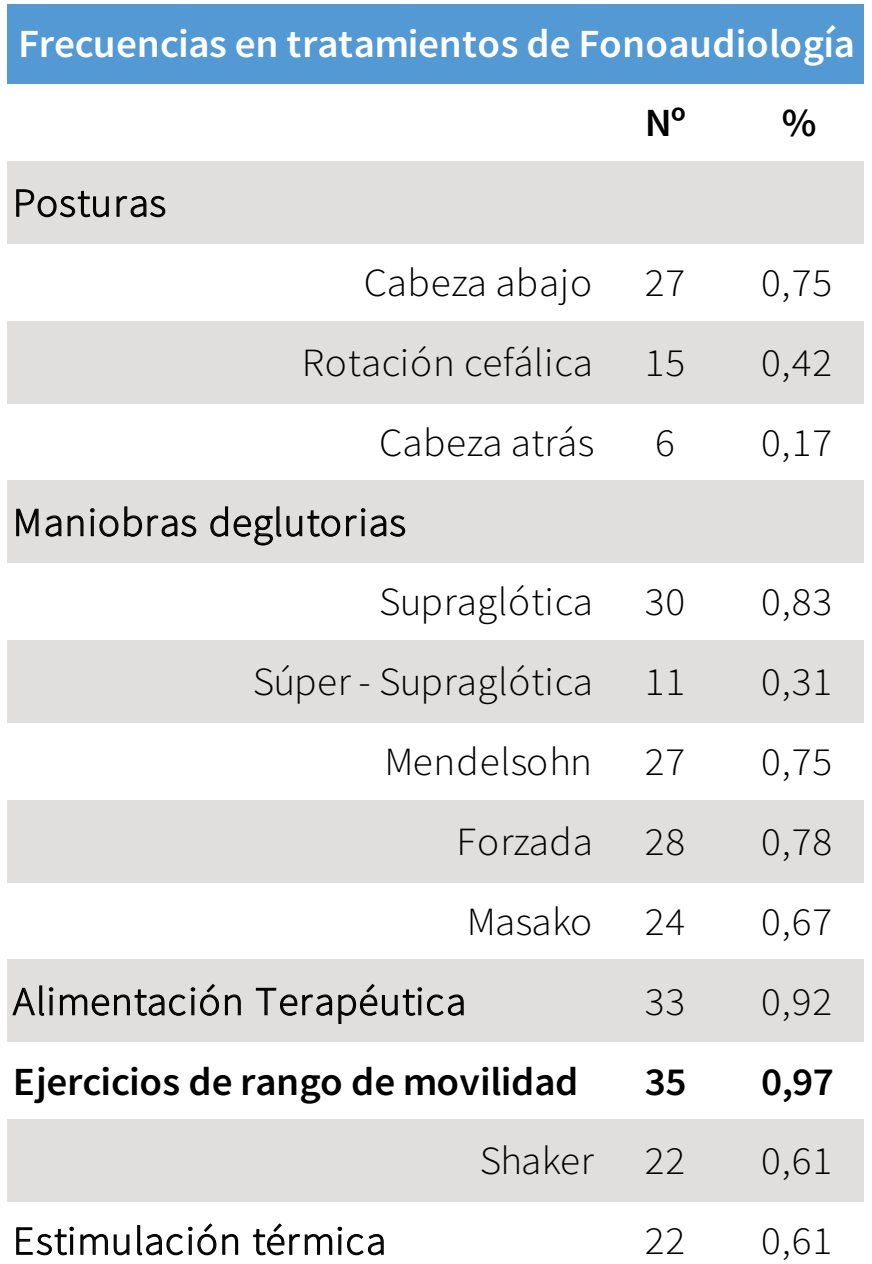

Fuente: Elaboración propia del autor.

En el análisis por test de $\chi^{2}$ se evidenció una correlación discreta de dependencia entre el sexo y el tipo de cáncer, el resultado del test $\chi^{2}$ fue 20.7, con un nivel de significancia $p<0,014$ para 9 grados de libertad lo cual, corrobora la hipótesis nula. Esta información se puede ver en la Tabla 4

Tabla 4. Correlación entre sexo y tipo de cáncer en una institución de Bogotá.

\section{$\chi^{2}$ Test (Sexo vs Tipo de cáncer)}

$\begin{array}{cccc} & \text { Value } & \text { df } & p \\ \chi^{2} & 20.7 & 9 & 0.014 \\ N & 36 & & \end{array}$

Fuente: Elaboración propia del autor.

Se observan resultados similares entre diferentes variables al realizar el test de $x^{2} y$ test de Fisher, el que obtuvo el valor $P$ menor fue el previamente descrito. No se evidencia asociación entre variables categóricas (edad dicotomizada por mediana vs resultado final de disfagia, entre otras); se necesita una muestra más grande para decretar asociaciones estadísticamente significativas.

\section{Discusión}

Es de gran importancia conocer e intervenir a tiempo los problemas de deglución secundarios al tratamiento de cáncer de cabeza y cuello para mejorar la calidad de vida y pronóstico de este tipo de pacientes (Lazarus, 2009). Combinando resultados de evaluaciones subjetivas, proporcionadas por el personal de la salud y por la percepción del pa- ciente, se tiene una visión más clara, exhaustiva y completa de la salud actual del paciente y su estado en el proceso de deglución.

Se demostró que el $64.28 \%$ de los pacientes durante tres meses presentaron diagnóstico subjetivo de disfagia, aunque algunos autores afirman que está alrededor del 80\% en todos los tipos de cáncer de cabeza y cuello (Denaro, Merlano, \& Russi, 2013) (Pauloski, 2008) (Frowen, Hughes, \& Skeat, 2019).

Por otro lado, los resultados de este estudio proporcionan información limitada del estado general de la prevalencia de disfagia orofaríngea en pacientes con cáncer de cabeza y cuello en una institución de cuarto nivel en Bogotá-Colombia, ya que el tamaño de la población no permitió generar mayores asociaciones estadísticas significativas entre las variables, sin embargo, es un estudio pionero en Colombia en el cálculo de la prevalencia de disfagia para el cáncer de cabeza y cuello.

En el análisis por test $\chi^{2} y$ Test de Fisher no se logró una correlación estadísticamente significativa para las variables analizadas, sin embargo, la que tuvo mayor aproximación fue sexo vs tipo de cáncer, lo cual está directamente relacionado con las estadísticas nacionales e internacionales y diferentes estudios de tratamiento (Cárcamo, 2018) (Liga contra el cáncer, 2016) (Siu, y otros, 2016).

En cuanto a la rehabilitación completa de la disfagia, el escaso número de pacientes que la lograron está, en gran medida, explicado por el hecho de dicotomizar la variable en "ausente" y "presente" usando como referencia la Escala Fols. Sin embargo, es de vital importancia aclarar que la mayoría de estos pacientes mejoraron en alguna medida su proceso deglutorio, aunque continuaban con algún grado de disfagia; y en algunos casos por el manejo médico-oncológico o por recidivas de la patología (Alvarez, y otros, 2007), su proceso de deglución inevitablemente iba en deterioro o se mantuvo

La modalidad de tratamiento asignada a cada paciente según el estadio de su enfermedad tiene efectos directos en la rehabilitación exitosa de la disfagia, evidenciado que a mayor cantidad de tratamientos existen menor cantidad de resultados positivos para la recuperación del proceso deglutorio. Efectos secundarios como la alteración estructural de la cirugía acompañados de la neurotoxicidad de la quimioterapia y la generación de tejido fibrótico de la radioterapia hacen que se produzca mayor acumulación del bolo, tanto en cavidad oral como a nivel retrofaríngeo por disminución de la sensibilidad, disminución del espacio faríngeo y limitación en la movilidad laríngea (Lazarus, Ward, \& Yiu, 2014), lo que aumenta el riesgo de broncoaspiración, disminuyendo la posibilidad de rehabilitación ya que en estos casos los efectos son irreversibles (Hutcheson, y otros, 2012).

Otro factor que influyó en la rehabilitación total de la disfagia fue el sistema de salud nacional que generó retrasos en la agenda de tratamientos para los pacientes (Suárez, Puerto-García, Rodríguez-Moreno, \& Ramírez-Moreno, 2017), permitiendo inicialmente diez sesiones con posibilidad de continuidad según indicación del profesional, para lo cual se debe reiniciar los trámites administrativos, lo que puede demorar entre dos y tres meses afectando índices de atención oportuna, lo cual concuerda con las medidas de tendencia central para esta variable. Además, se presentaron situaciones económicas, dificultad en desplazamientos y baja adherencia a los tratamientos que llevan a la deserción del proceso terapéutico. Por tal razón, se evidencian valores extremos en el número de sesiones que tomó cada uno de los pacientes durante este periodo.

Para la rehabilitación de la disfagia se utilizan técnicas directas e indirectas seleccionadas según el caso de cada paciente, se evidenció que las más utilizadas son los ejercicios de rangos de movilidad teniendo en cuenta que, en la mayoría de los casos, secundario a la radiotera- 
pia los rangos de movilidad cervical y de cintura escapular disminuyen lo que genera cambios morfológicos en el posicionamiento laríngeo y, por ende, en la deglución. Otra de las técnicas usadas con alta frecuencia en la alimentación terapéutica que se hace necesario ya que es el proceso en sí, y dentro de las maniobras deglutorias como tal tuvo mayor efectividad la supraglótica que en varios casos fue modificada sin alterar su objetivo de lograr estabilidad laríngea y cierre de la vía aérea durante la deglución (Pauloski, 2008) (Cámpora \& Falduti, 2012) (Wheeler-Hegland, y otros, 2009)

\section{Conclusión}

El manejo oncológico en cáncer de cabeza y cuello genera consecuencias dentro de los procesos que involucran estas estructuras, uno de los procesos más significativos es la deglución. Durante el procedimiento quirúrgico y la quimio-radioterapia se generan cambios en la actividad muscular y nerviosa que lleva a alteraciones en la coordinación y agilidad en la movilidad de los órganos del sistema estomatognático, esto se evidencia en la dificultad presentada durante la alimentación manifestada en signos de disfagia y desnutrición.

Teniendo en cuenta la alta prevalencia de la disfagia secundaria a tratamiento de cáncer de cabeza y cuello, se requiere de una intervención oportuna con un equipo multidisciplinario en donde el fonoaudiólogo desempeña un papel importante, la evaluación- diagnóstico permite determinar la presencia de signos de disfagia, determinar la seguridad y eficacia de la vía oral y pronóstico deglutorio; además permite enfocar el tratamiento hacía la recuperación de la actividad muscular normal o hacia la compensación, teniendo en cuenta que las cirugías de cabeza y cuello en su mayoría son reconstructivas.

En cuanto al tratamiento, con base en los resultados de la evaluación, el asertividad en el manejo, combinación y adaptación de técnicas compensatorias y facilitadoras a cada paciente va a permitir la rehabilitación de la vía oral siempre enfocada hacia la funcionalidad. Esta evolución va sujeta a factores relacionados como estadio y tipo de tratamiento del cáncer, pronóstico oncológico, edad, continuidad del tratamiento y disponibilidad del paciente. La pertinencia y oportunidad en la toma de decisiones con respecto a la seguridad de la vía oral y el asertividad en la aplicación de técnicas de tratamiento le van a permitir una intervención más efectiva.

Se sugieren estudios con una $N$ mayor que permita correlacionar significativamente mayor cantidad de variables y respondan al contexto colombiano, para determinen el tiempo de rehabilitación y las técnicas de tratamiento más eficaces según el tipo de cirugía y reconstrucción y compararlas con los datos estadísticos que se presentan a nivel mundial.

\section{Referencias}

Alvarez, C., Llorente, J., Gutierrez, V., Fernandez, H., Guervos, M., Suarez, C., \& Hermen, M. (2007). Recidivas en el cáncer escamoso de cabeza y cuello. Acta Otorrinolaringológica Española, 58(4), 156-163. doi:DOl: 10.1016/S0001-6519(07)74902-6

American Cancer Society, NHC. (2015). Recuperado el 5 de Octubre de 2016, de Una guía sobre Radioterapia: http://www.cancer.org/acs/groups/ cid/documents/webcontent/003027-pdf.pdf
American Cancer Society, NHC. (2015). American Cancer Society. Recuperado el 5 de Octubre de 2016, de Guía sobre Radioterapia: https://www.cancer.org/es/tratamiento/tratamientos-y-efectossecundarios/tipos-de-tratamiento/radioterapia.html

Cadena, E., Guerra, R., \& Pérez-Mitchell, C. (Julio-Septiembre de 2014). Cirugía Robótica Transoral (TORS), en el manejo de lesiones neoplásicas de cabeza y cuello. Revista Colombiana de Cancerología, 18(3), 128-136. doi:10.1016/j.rccan.2014.06.003

Cadena, E., Guerra, R., \& Pérez-Mitchell, C. (Julio-Septiembre de 2014). Cirugía Robótica Transoral (TORS), en el manejo de lesiones neoplásicas de cabeza y cuello. Revista Colombiana de Cancerología, 18(3), 128-133. doi:https://doi.org/10.1016/j. rccan.2014.06.003

Cámpora, H., \& Falduti, A. (2012). Evaluación y tratamiento de las alteraciones de la deglución. Revista Americana de Medicina Respiratoria, 3(1), 98-107.

Cárcamo, M. (Ago de 2018). Epidemiología y generalidades del tumor de cabeza y cuello. Revista Médica Clínica Las Condes, 29(4), 387-492. doi:DOI: 10.1016/.rmclc.2018.06.009

Denaro, N., Merlano, M., \& Russi, E. (Sep de 2013). Dysphagia in Head and Neck Cancer Patients: Pretreatment Evaluation, Predictive Factors, and Assessment during Radio-Chemotherapy, Recommendations. Clinical and Experimental Otorhinolaryngology., 6(3), 117126. doi:https://www.e-ceo.org/journal/view.php?doi=10.3342/ ceo.2013.6.3.117

European Society for Medical Oncology. (01 de 01 de 2015). Fundación contra el cáncer. Recuperado el 18 de 06 de 2019, de ESMO: ESMOACF-Cancer-de-Cabesa-y-Cuello-Guia-para-pacientes.pdf

Frowen, J., Hughes, R., \& Skeat, J. (2019). The prevalence of patientreported dysphagia and oral complications in cancer patients. Supportive Care in Cancer, 1-10. doi:doi: 10.1007/s00520-019-04921-y

Greenblatt, D., Sippel, R., Leverson, G., Frydman, F., Schaefer, S., \& Chen, H. (2009). Thyroid Resection Improves Perception of Swallowing Function. World Journal of Surgery, 33, 255-260. doi:10.1007/500268008-9837-9

Greenblatt, D., Sippel, R., Leverson, G., Frydman, F., Schaefer, S., \& Chen, H. (2009). Thyroid Resection Improves Perception of Swallowing Function. World Journal of Surgery, 33, 255-260. doi:https://doi. org/10.1007/s00268-008-9837-9

Hamilton, S., Yoo, J., Hammond, A., Read, N., Venkatesan, V., Franklin, J., ... Badhwar, A. (Oct de 2008). Microvascular changes in radiationinduced oral mucositis. J Otolaryngol Head Neck Surg, 37(5), 730-737.

Hutcheson, K., Lewin, J., Barringer, D., Lisec, A., Gunn, B., Moore, M., \& Holsinger, C. (2012). Late Dysphagia After Radiotherapy-Based Treatment of Head and Neck Cancer. Cancer, 118(23), 5793-5799. doi:https://doi.org/10.1002/cncr.27631

Joung, S., Woo, K., Beom, S., Hwa, J., \& Kyu, M. (Oct de 2015). Voluntary Cough and Swallowing Function Characteristics of Acute Stroke Patients Based on Lesion Type. Archives of Physical Medicine and Rehabilitation, 96(10), 1866-1872. doi:https://doi.org/10.1016/j. apmr.2015.06.015

Lazarus, C. (Jun de 2009). Effects of chemoradiotherapy on voice and swallowing. Current Opinion in Otolaryngology \& Head Neck Surgery, 17, 172-178. doi:10.1097/M00.0b013e32832af12f.

Lazarus, C., Ward, E., \& Yiu, E. (2014). Speech and Swallowing following oral, oropharyngeal and nasopharyngeal cancers. En E. Ward, \& C. Van As-Brooks, Head and Neck Cancer: Treatment, Rehabilitation, and Outcomes (págs. 173-240). San Diego, California: Plural Publishing.

Liga contra el cáncer. (01 de 01 de 2016). Liga contra el cáncer seccional Bogotá. Recuperado el 18 de 06 de 2019, de http://www. ligacontraelcancer.com.co/cancer-de-cabeza-y-cuello/ 


\section{Prevalencia de la disfagia secundaria al tratamiento de cáncer de cabeza y cuello}

\section{En una cohorte de una institución en bogotá}

Liga Contra el Cáncer. (01 de 01 de 2016). Liga contra el cáncer seccional Bogotá. Recuperado el 18 de 06 de 2019, de http://WWW. ligacontraelcancer.com.co/cancer-de-cabeza-y-cuello/

Logemann, J., Pauloski, B., Rademaker, A., \& Colangelo, L. (1 de Mayo de 1997). Speech and swallowing rehabilitation for head and neck cancer patients. Oncology (Williston Park), 11(5), 659-664.

Lyndell, K. (2014). Radiation and Chemotherapy. En E. Ward, \& C. Van as-brooks, Head and neck cancer: treatment, rehabilitation, and outcomes (Second Edition ed., págs. 63-102). San Diego, California: Plural Publishing.

Martínez, M., \& Bellido, D. (2003). Nutrición y disfagia. Fisioterapia, 25(5), 306-310. doi:https://doi.org/10.1016/S0211-5638(03)73069-7

Nickel, B., Tan, T., Cvejic, E., Baade, P., McLeod, D., Pandeya, N., ... Jordan, S. (17 de 01 de 2019). Health-Related Quality of Life After Diagnosis and Treatment of Differentiated Thyroid Cancer and Association With Type of Surgical Treatment. JAMA Otolaryngology-Head \& Neck Surgery, e1-e8. doi:10.1001/jamaoto.2018.3870.

Nickel, B., Tan, T., Cvejic, E., Baade, P., McLeod, D., Pandeya, N., .. J Jordan, S. (17 de jan de 2019). Health-Related Quality of Life After Diagnosis and Treatment of Differentiated Thyroid Cancer and Association With Type of Surgical Treatment. JAMA Otorinolaryngology - Head \& Neck Surgery, 231-238. doi:doi:10.1001/jamaoto.2018.3870

Pauloski, B. (Nov de 2008). Rehabilitation of Dysphagia Following Head and Neck Cancer. Physical Medicine and Rehabilitation Clinic of North America, 19(4), 889-928. doi:doi: 10.1016/j.pmr.2008.05.010.

Portas, J., Pereira, C., Perissato, E., Dos Santos, D., Sampaio, A., Aparecido, R., \& Brandao, A. (Ene-Feb de 2011). Swallowing after non-surgical treatment (radiation therapy / radiochemotherapy protocol) of laryngeal cancer. Brazilian Journal of Otorhinolaryngology, 77(1), 96-101.

Santini, L., Robert, D., Lagier, A., Dessi, P., \& Fakhry, N. (Junio de 2015). A videofluoroscopic study comparing severe swallowing disorders in patients treated surgically or with radiation for oropharyngeal cancer. International Journal of Oral and Maxillofacial Surgery, 44(6), 705-709. doi:https://www.ijoms.com/article/S0901-5027(15)00044-2/ fulltext
Shaw, S., \& Martino, R. (Dec de 2013). The normal swallow: muscular and neurophysiological control. Otolaryngology Clinics of North America, 46(6), 937-956. doi:10.1016/j.otc.2013.09.006.

Siu, L., Waldron, J., Chen, B., Winquist, E., Wright, J., Nabid, A., ... Johnson, A. (Dic de 2016). Effect of Standard Radiotherapy With Cisplatin vs Accelerated Radiotherapy With Panitumumab in Locoregionally Advanced Squamous Cell Head and Neck Carcinoma: A Randomized Clinical Trial. JAMA oncology, 3(2), 220-226. doi:doi: 10.1001/ jamaoncol.2016.4510.

Smeele, L. (2014). Oral, oropharyngeal and nasopharyngeal cancer: intervention approaches. En E. Ward, \& C. Van As-Brooks, Head and neck cancer: treatment, rehabilitation, and outcomes (ed., págs. 103-120). San Diego, California: Plural Publishing.

Suárez, L., Puerto-García, S., Rodríguez-Moreno, L., \& Ramírez-Moreno, J. (2017). La crisis del sistema de salud colombiano: una aproximación desde la legitimidad y la regulación. Revista Gerencia y Políticas de Salud, 16(32), 34-50. doi:https://doi.org/10.11144/Javeriana.rgps16-32. CSSC

Szczesniak, M., Maclean, J., Zhang, T., Graham, P., \& Cook, I. (Nov de 2014). Persistent Dysphagia after Head and Neck Radiotherapy: A Common and Under-reported Complication with Significant Effect on Non-cancer-related Mortality. Clinical Oncology (Royal College of Radiologist), 26(11), 697-703. doi:doi: 10.1016/j.clon.2014.08.009

Wheeler-Hegland, K., Ashford, J., Frymark, T., McCabe, D., Mullen, R., Musson, N., ... Schooling, T. (2009). Evidence-based systematic review: Oropharyngeal dysphagia behavioral treatments. Part IIImpact of dysphagia treatment on normal swallow function. Journal of rehabilitation research and development, 46(2), 185-194.

Zhang, L., Huang, Z., Wu, H., Chen, W., \& Huang, Z. (Dic de 2014). Effect of swallowing training on dysphagia and depression in postoperative tongue cancer patients. European Journal on Oncology Nurse, 18(6), 626-629. doi:10.1016/j.ejon.2014.06.003 